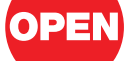

SUBJECT AREAS: PHYSICS

OPTICAL PHYSICS

ULTRAFAST PHOTONICS

METAMATERIALS

Received

15 June 2012

Accepted

25 March 2013

Published

9 April 2013

Correspondence and requests for materials should be addressed to

G.Z. Igazheng@ caltech.edu) or L.R. (ranlx@zju.edu.cn)

\section{Negative Group Velocity in the Absence of Absorption Resonance}

\author{
Dexin Ye', Guoan Zheng'2, Jingyu Wang', Zhiyu Wang', Shan Qiao³, Jiangtao Huangfu' \& Lixin Ran'
}

\author{
'Laboratory of Applied Research on Electromagnetics (ARE), Zhejiang University, Hangzhou, 310027, China, ${ }^{2}$ Department of \\ Electrical Engineering, California Institute of Technology, Pasadena, CA, 91125 , USA, ${ }^{3}$ Zhejiang University City College, \\ Hangzhou, 310015, China
}

Scientific community has well recognized that a Lorentzian medium exhibits anomalous dispersion behavior in its resonance absorption region. To satisfy the Krammers-Kronig relation, such an anomalous region has to be accompanied with significant loss, and thus, experimental observations of negative group velocity in this region generally require a gain-assisted approach. In this letter, we demonstrate that the negative group velocity can also be observed in the absence of absorption resonance. We show that the $\mathrm{k}$-surface of a passive uniaxial Lorentzian medium undergoes a distortion near the plasma frequency. This process yields an anomalous dispersion bandwidth that is far away from the absorption resonance region, and enables the observation of negative group velocity at the plasma frequency band. Introducing anomalous dispersion in a well-controlled manner would greatly benefit the research of ultrafast photonics and find potential applications in optical delay lines, optical data storage and devices for quantum information processing.

n electromagnetics, the classical Drude-Lorentz model offers an intuitive and accurate description of both normal and anomalous material dispersion in an extended spectrum from direct current to optical frequencies $^{1,2}$. As a direct consequence of the Kramers-Kronig relation, anomalous dispersion occurs as the wavelength approaches to the electric scale of the absorption resonance of the medium ${ }^{3}$, exhibiting a reversed spatial-temporal frequency dependence in the opposite of the normal dispersion case. Without violating the causality $^{4-7}$, anomalous dispersion in the absorption resonance region implies an electromagnetic (EM) wave packet propagating with a superluminal or negative group velocity, i.e., an EM pulse appears to exit the medium before it enters ${ }^{8-17}$. In this paper, instead, we demonstrate that the negative group velocity can also be observed in the absence of absorption resonance. We show that when an EM wave is incident obliquely upon the surface of a uniaxial medium with a Lorentzian dispersion along its optical axis, the k-surface of the refractive wave undergoes a distortion as the frequency sweeps across the plasma frequency. Such a process yields an anomalous dispersion bandwidth that is far away from the absorption resonance region, and enables a new scheme for dispersion engineering in a well-controlled manner. This paper is structured as follows. We will first show the phase matching analysis of the proposed approach; next, we will report on our full wave simulation results. We will then report on our experimental observation of the negative group velocity at the plasma frequency band. Finally, we will draw our conclusion at the end of this paper.

\section{Results}

Phase matching analysis. The group velocity describing the propagation of wave packets in an isotropic medium can be expressed as $v_{g}=c / n_{g}$, where $n_{g}=n+\omega \cdot d n / d \omega, n$ is the usual refractive index, $c$ is the speed of light in vacuum and $\omega$ is the angular frequency. In the anomalous dispersion region with strong absorption resonance, the term $d n / d \omega$ in $n_{g}$ is negative, and as such, the group velocity can be a negative value if this term is sufficiently small. This is exactly the phenomenon that has been reported previously ${ }^{4,8-17}$. Normally, this phenomenon does not occur at the plasma frequency band, which is a normal-dispersive region in the Drude-Lorentz model. However, the situation is different when the medium is anisotropic ${ }^{18,19}$. Recently, an optical topological transition in a strongly anisotropic metamaterials has been described ${ }^{20,21}$. In this paper, we further point out that during such topological transition, the k-surface undergoes a distortion. This process yields an anomalous dispersion bandwidth and enables the observation of negative group velocity at the plasma frequency band.

Without losing generality, we begin with a simplest uniaxial medium with a scalar permittivity $\varepsilon_{r}=1$ and a permeability tensor $\overline{\bar{\mu}}_{r}=\operatorname{diag}\left(1,1, \mu_{r z}\right)$, where $\mu_{r z}=1-\omega_{p}^{2} /\left[\omega\left(\omega+i \gamma_{e}\right)\right]$ follows a frequency dependence of Drude model with a plasma frequency $\omega_{p}$ and a damping frequency $\gamma_{e}$. Notice that all of above constitutive components 
are normal dispersive and, unlike the absorption resonance case, the loss of $\mu_{r z}$ characterized by $\gamma_{e}$ can be very small, as indicated later.

As shown in Fig. 1a, with an oblique EM incidence from the air (electric field polarized along the y direction) and a wave vector $\bar{k}=$ $\hat{x} k_{x}+\hat{z} k_{z}$, the dispersion relation (or k-surface) in the medium is

$$
k_{x}^{2} / \mu_{r z}+k_{z}^{2}=\varepsilon_{r} k_{0}^{2}=\varepsilon_{r}(\omega / c)^{2},
$$

where $k_{0}$ is the wave number in air, $k_{x}$ and $k_{z}$ are wave numbers in $\mathrm{x}$ and $\mathrm{z}$ directions, respectively. Based on the phase matching condition, for a given incident angle $\theta$, we have $k_{x}=k_{0} \sin \theta$ and $k_{z}= \pm \sqrt{\varepsilon_{r}(\omega / c)^{2}-k_{x}^{2} / \mu_{r z}}$. Due to the passivity nature of the discussed medium, the sign of $k_{z}$ can be determined by choosing the $k_{z}$ with a positive imaginary part.

For a specified damping frequency, the shape of the k-surface described by equation (1) would strongly depend on the sign of $\mu_{r z}$, as shown in Fig. 1b. In a hypothetical lossless case shown in the upper panel of Fig. 1b (the damping frequency is set to 0 ), the k-surface could be either a closed ellipse $\left(\omega>\omega_{p}, \mu_{r z}>0\right)$ or an open, two-branch hyperbola $\left(\omega>\omega_{p}, \mu_{r z}<0\right)$, and at the exact plasma frequency with $\mu_{r z}=0$, the ellipse and the hyperbola shrink separately to a line segment $\left(\overline{F_{1} F_{2}}\right.$, when $\left.\omega \rightarrow \omega_{p}+\right)$ and two rays $\left(\overline{F_{1} R_{1}}\right.$ and $\overline{F_{2} R_{2}}$, when $\left.\omega \rightarrow \omega_{p}-\right)$ on the $\mathrm{z}$ axis. Therefore, under the lossless hypothesis, there is a discontinuity of the k-surface at the plasma frequency: when the frequency sweeps across the plasma frequency, the k-surface changes abruptly from a line segment to two rays, which breaks the continuity of dispersion and implies the non-causality of the lossless hypothesis ${ }^{22}$. On the other hand, when the damping frequency is not zero (shown in the lower panel of Fig. 1b), the k-surface distorts from a closed ellipse to an open, two-branch curve that looks like a hybrid of an ellipse and a hyperbola. The continuous evolving of the k-surface from a distorted ellipse to a distorted hyperbola implies the causality and physical reliability of such a Lorentzian medium.

Next we focus our discussion on the continuous distortion of the $\mathrm{k}$-surface at the vicinity of the plasma frequency. In Fig. 1c, we show the phase matching diagram for two adjacent frequencies ( $\omega$ and $\omega$ $+\delta$ ) in the transition zone (i.e. around the plasma frequency) of the $\mathrm{k}$-surface distortion. We define a critical angle $\theta_{c}$ (see Supplementary Note 1) for the discussion of the following two cases: 1) $\theta_{1}<\theta_{c}$, an inclined EM wave propagates at the forward direction with a group velocity of $v_{g 1}$;2) $\theta_{2}>\theta_{c}$, an inclined EM wave propagates at the backward direction with a group velocity of $v_{g 2}$. We also note that, the phase velocities denoted by $v_{p 1}$ and $v_{p 2}$ remain positive, meaning that the phase velocities of the carriers always propagate forwardly in both cases. Therefore, the negative group velocity $v_{g 2}$ is not accompanied with a negative refraction. From equation (1), both $v_{g 1}$ and $v_{g 2}$ can be calculated by

$$
\bar{v}_{g}=\nabla_{\bar{k}} \omega(\bar{k})=\hat{x} v_{g x}+\hat{z} v_{g z}=\hat{x} \frac{1}{\operatorname{Re}\left[d k_{x} / d \omega\right]}+\hat{z} \frac{1}{\operatorname{Re}\left[d k_{z} / d \omega\right]} .
$$

In the rest of the paper, we focus our discussions on the group velocity at the propagating direction, i.e. the $\mathrm{z}$ direction. As shown in Fig. 1d, for a smaller incident angle $\theta_{1}$ (green curves), $k_{z}$ is normaldispersive with a small loss term denoted by the dash green curve; while, for a larger angle $\theta_{2}, k_{z}$ exhibits an anomalous dispersion behavior inside the transition zone (marked in grey, also see Supplementary Note 2). We note that, regardless the normal-dispersive property of $\mu_{r z}$ (the inset of Fig. 1d), the dispersion of $k_{z}$ is anomalous, and the behavior of $k_{z}$ in this transition zone is similar to that in the conventional absorption resonance region.

Full wave simulation. We performed a full wave EM simulation based on the time domain propagation of wave packets using a a

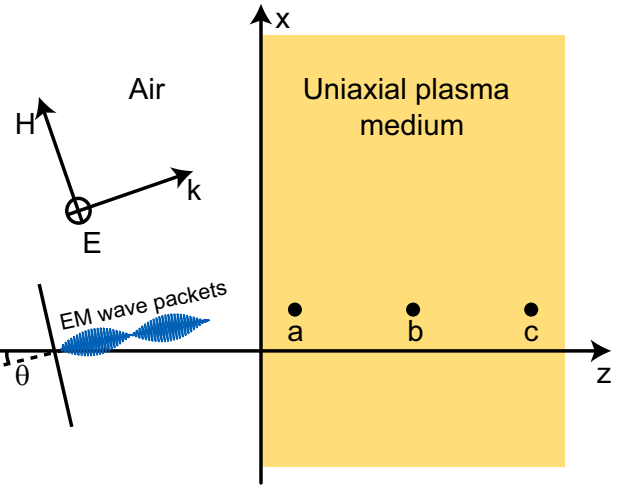

C

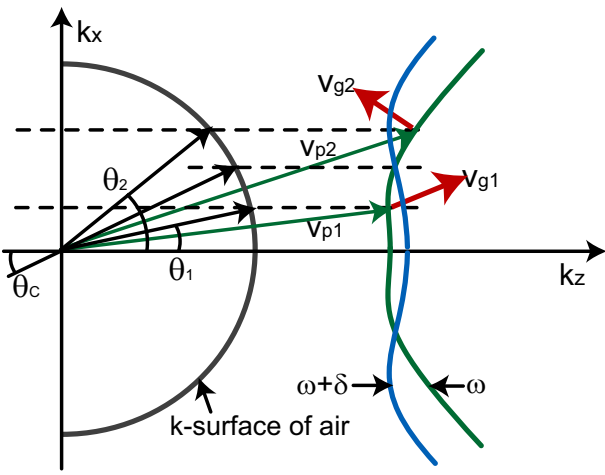

b

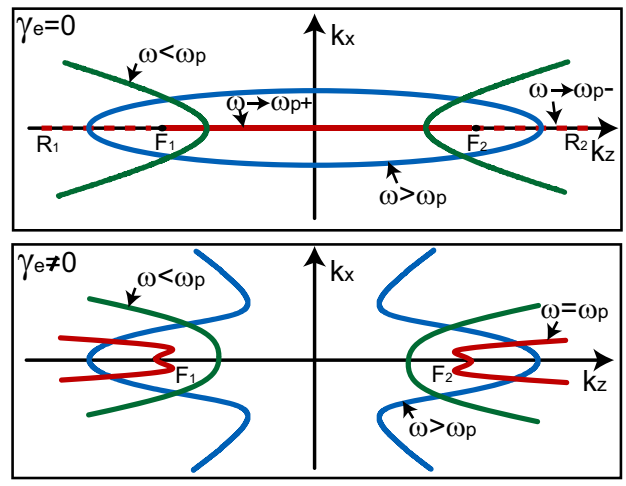

d

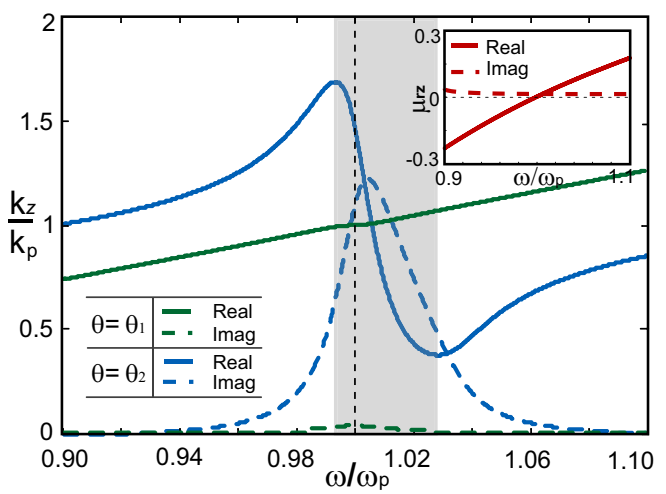

Figure 1 Theoretical analysis. (a) Diagram of EM wave packets impinge on the surface of a uniaxial plasma medium. (b) Illustration of the k surfaces around plasma frequency in cases with (bottom) and without loss (top). (c) Phase matching at the boundary between air and the discussed medium. (d) Dispersion of $k_{z}$ for different incident angles, where $k_{p}=\omega_{p} / c$, Inset shows the dispersion of $\mu_{r z}$ in the same frequency zone, where $\gamma_{e}$ is assumed to be $0.02 \omega_{p}$. 

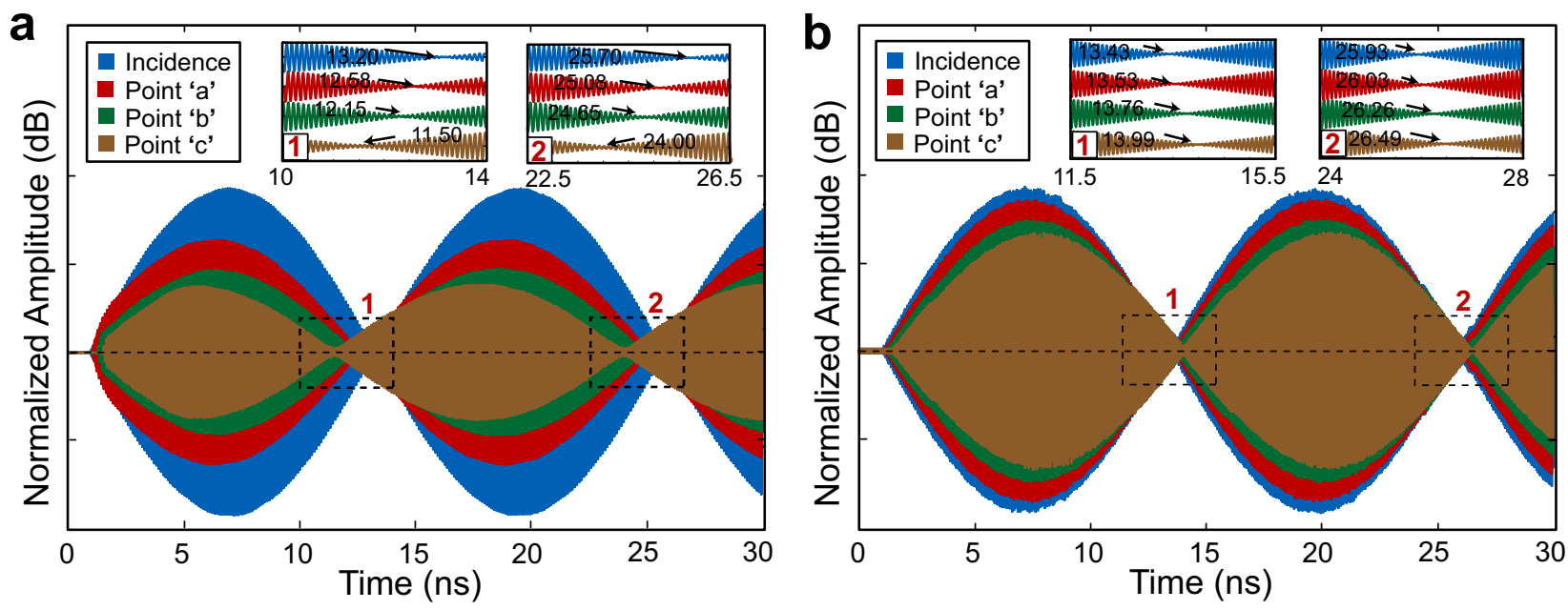

Figure $2 \mid$ Full wave simulation of group velocities in a uniaxial plasma medium with $\omega_{p}=10 \mathrm{GHz}$ and $\gamma_{\mathrm{e}}=0.02 \omega_{p}$ for different carrier frequencies. (a) The frequency of carrier is $10 \mathrm{GHz}$ and (b) The frequency of carrier is $12 \mathrm{GHz}$, where the incident angle is $10^{\circ}$. The blue curve denotes the detected incident EM packets at the surface of the discussed medium. The amplitude of each waveform is normalized by the incident wave.

commercial software package, CST microwave studio. As shown in Fig. 1a, we recorded the signals of the wave packets in a 20 -cm-thick magnetically uniaxial medium. The optical axis of this medium is at the $\mathrm{z}$ direction and the dispersion of $\mu_{r z}$ follows the Drude model, with $\omega_{p}=10 \mathrm{GHz}$ and $\gamma_{e}=0.02 \omega_{p}$. The incident angle was chosen to be 10 degrees, larger than the critical angle $\theta_{c}$ which is determined to be 1.8 degrees under this configuration. In order to measure the signal amplitudes inside the medium, three probes were placed at point ' $a$ ', 'b' and 'c', with a 70-mm increment, as shown in Fig. 1a.

In this case, the transition zone of this configuration is estimated to be $9.93-10.22 \mathrm{GHz}$ (see Supplementary Note 2). Two simulations were performed for frequencies both inside (Fig. 2a) and outside (Fig. 2b) the transition zone. In the first simulation, a 10-GHz carrier modulated with a $40-\mathrm{MHz}$ sinusoidal envelope signal was simulated as the incident wave. The detected signals at the three points, as well as the incident wave at the surface of the medium, are shown in Fig. 2a. Several distinct features are worth noting: 1) due to the imaginary part of $k_{z}$ in Fig. 1d, the signal intensity decays with an attenuation rate of $-1.28 \mathrm{~dB} / \mathrm{cm}$ from point 'a' to point ' $c$ '; 2 ) for the first wave packet, the 'front velocity' of the envelope signal is positive $^{23}$; 3) except the front knot of the first packet, all the successive peaks and knots are first seen at the furthest point ' $c$ ' (see the magnified panels labeled with ' 1 ' and ' 2 '), implying that they propagate backwardly from point ' $c$ ' to point ' $a$ '; 4) Compared with the incident wave measured at the surface (blue line), the wave front of the second wave packet arrives at point ' $c$ ' even before the first wave packet entirely enters into the medium, implying the existence of the negative group velocity. We note that, despite the fact of the negative group velocity, the front velocity of the signal is always positive, in other words, the information velocity carried by the wave front is still positive. As a comparison, we performed the second simulation with a carrier frequency of $12 \mathrm{GHz}$ in Fig. $2 \mathrm{~b}$ as a reference. In this case, the frequency is not in the transition zone (i.e. normal-dispersive behavior is expected). It is seen that the front and group velocities are all positive in Fig. $2 b$.

Experimental verification. We demonstrated the experimental verification of our analysis based on the concept of metamaterial ${ }^{24}$. Consisting of densely arranged artificial, sub-wavelength electric and/or magnetic dipole moments, metamaterials are capable of providing predefined effective constitutive parameters based on the Maxwell-Garnett effective medium theory ${ }^{25,26}$. Fig. 3a shows the slablike metamaterial sample. Consisting of multilayer printed circuit boards carrying periodically arranged sub-wavelength split-ring resonators (SRRs), the dispersion of the effective z-component permeability $\left(\mu_{r z}\right.$, shown in the lower inset) closely follows a a

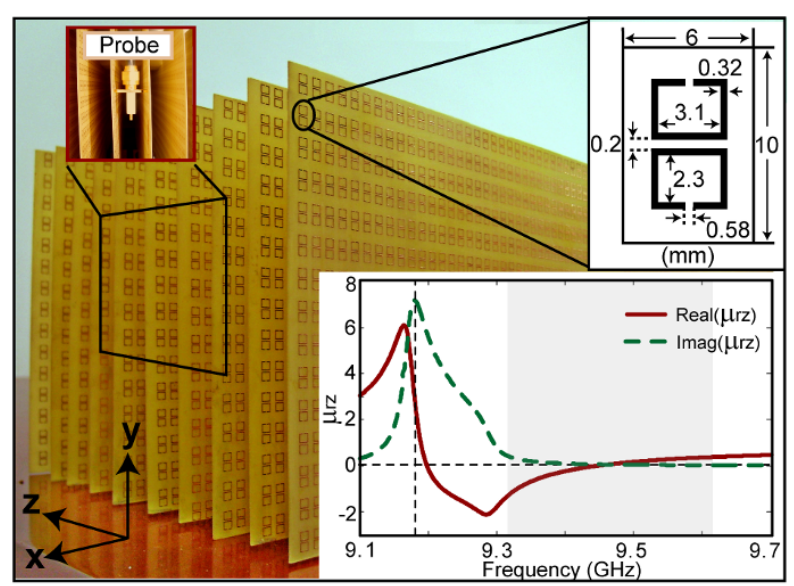

b

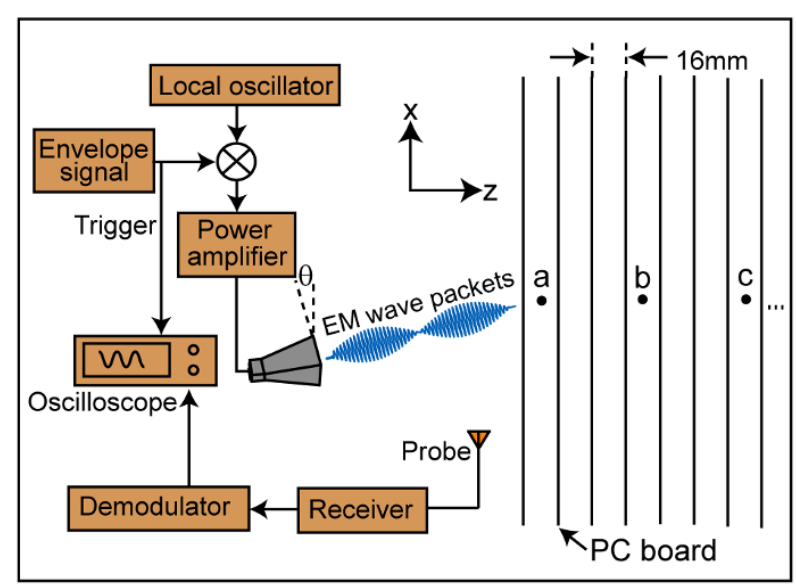

Figure 3 Experimental structure and setup. (a) Metamaterial sample and the real and imaginary parts of $\mu_{r z}$ retrieved from simulation data. (b) Experimental setup. 
typical Drude-Lorentz dispersion model with an absorption resonance around $9.19 \mathrm{GHz}$ and a typical plasma dispersion region between $9.35 \mathrm{GHz}$ and $9.65 \mathrm{GHz}$ (marked in gray, with $\omega_{p} \approx 9.45 \mathrm{GHz}$ and a small imaginary part varying from 0.02 to 0.2 ). Fig. $3 \mathrm{~b}$ shows the experimental setup for generating and detecting the EM wave packets. Detailed information of the metamaterial sample and the experimental setup are provided in Methods.

With a coherence measurement of the transmitting wave packets inside the sample, the demodulated envelope signals with the carrier frequency of $9.5 \mathrm{GHz}$ (in the transition zone) and $10 \mathrm{GHz}$ (out of the transition zone) were directly obtained, as shown in Fig. $4 \mathrm{a}$ and $4 \mathrm{~b}$. In
Fig. 4a, the amplitude of envelope decays as the wave propagates from point 'a' to point ' $c$ ' with an attenuation rate of $-2.18 \mathrm{~dB} / \mathrm{cm}$, corresponding to a propagation length of $1.99 \mathrm{~cm}$. On the other hand, the knots (as well as the peaks) of wave envelop are first seen at the furthest point ' $c$ ', and they propagate backwardly to point ' $b$ ' (1.51 ns later) and then point 'a' (2.57 ns later). As a comparison, in the $10-\mathrm{GHz}$ carrier case (outside the transition zone), the knots of wave envelop propagate from nearest point ' $a$ ' to point ' $b$ ' ( 0.83 ns later) and then point ' $c$ ' (1.58 ns later) with an attenuation rate of $-0.16 \mathrm{~dB} / \mathrm{cm}$, corresponding to a propagation length of $27 \mathrm{~cm}$. Fig. $4 \mathrm{c}$ shows the measured group delay $\Delta t$ (upper panel), the attenuation rate (lower panel) versus carrier frequency in the transition zone
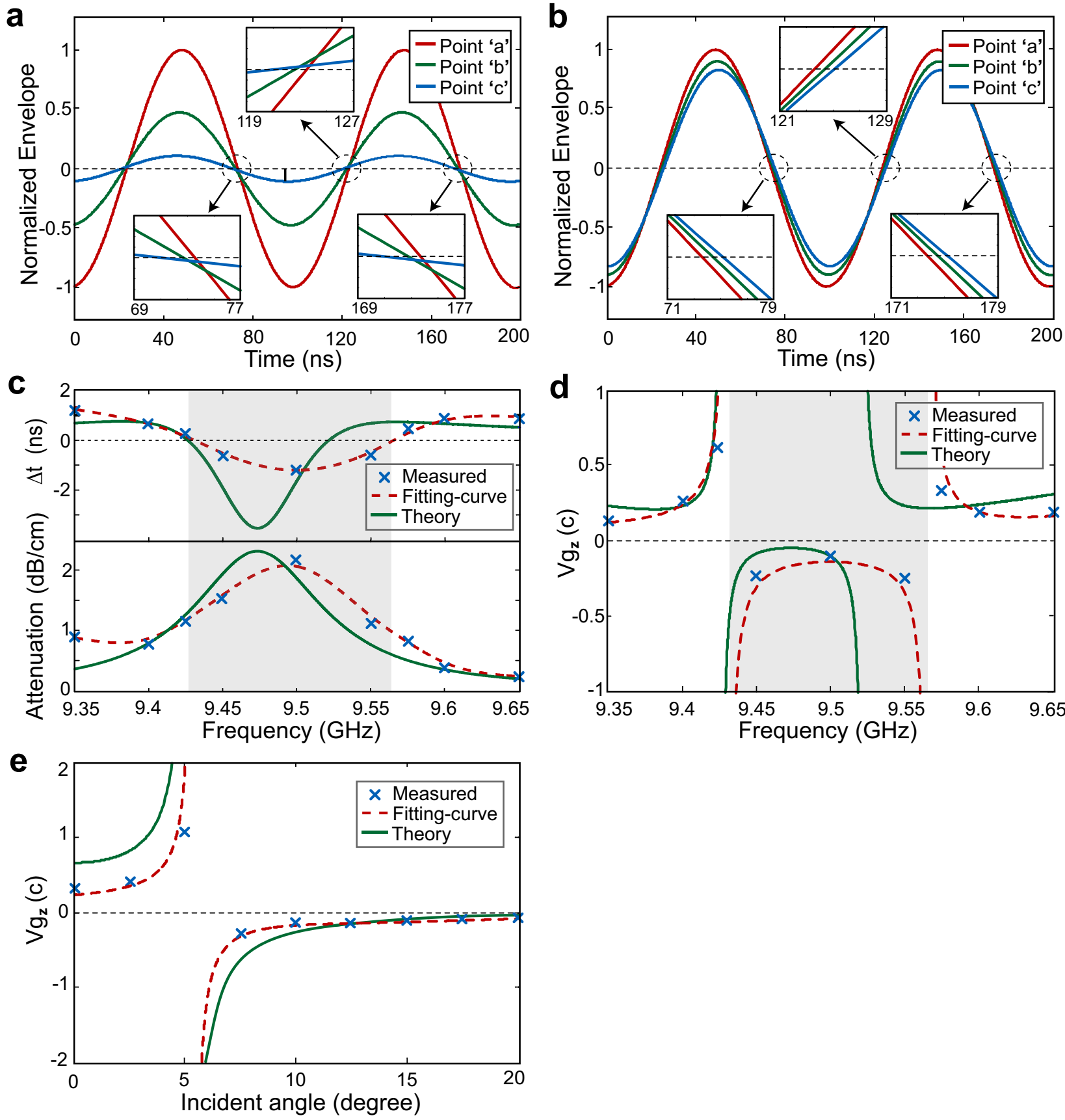

Figure $4 \mid$ Experimental results and analysis. (a) The demodulated envelope signals in time domain measured at points 'a', 'b' and 'c', where the frequency of the carrier is $9.5 \mathrm{GHz}$. (b) The same measurement when the frequency of carrier is $10 \mathrm{GHz}$. (c) The measured and theoretical group delay $\Delta t$ (upper panel) and attenuation (lower panel) versus carrier frequencies in the transition zone with an incident angle of 15 degrees. (d) The measured and theoretical group velocity $v_{g z}$ versus carrier frequencies. (e) The measured and theoretical group velocity $v_{g z}$ versus different incident angles at 9.5 GHz. 
with an incident angle of 15 degrees. For comparison, we also show in Fig. $4 \mathrm{c}$ the group delay and attenuation rate calculated by equation (2) with the simulation-based $\varepsilon_{r}$ and $\mu_{r z}$ shown in Fig. 3a. We see that both experimental results of group delay and attenuation rate are consistent with the trend of the theoretical calculations, except for a slight difference in central frequency and bandwidth. Comparing with the experimental results, the calculated bandwidth is $40 \mathrm{MHz}$ narrower with a frequency shift of $30 \mathrm{MHz}$. This slight discrepancy can be attributed to the difference between the actual and simulationbased $\varepsilon_{r}$ and $\mu_{r z}$. It is well known that the loss of a fabricated metamaterial is higher than the simulation case due to the misalignment of unit cells and the incontrollable machining errors (In Supplementary Note 3, it is shown that either a higher material loss or a larger incident angle leads to the increase of the bandwidth of the transition zone).

In Fig. $4 \mathrm{~d}$, the $\mathrm{z}$ component of the group velocity is calculated by $v_{g z}=\Delta d / \Delta t$, where $\Delta d$ is the distance between point ' $\mathrm{b}$ ' and point ' $\mathrm{c}$ ', i.e., $48 \mathrm{~mm}$. Notice that the group velocities marked in blue dots, dashed red line and solid green line are calculated from the corresponding points and curves in Fig. $4 \mathrm{c}$. As a direct consequence, $v_{g z}$ is negative in the transition band. The calculated curves also show that $v_{g z}$ approaches to negative infinity at the edges of the transition zone, reflecting the fact that the group delay $\Delta t$ continues at zero when $v_{g z}$ changes its sign. Fig. $4 \mathrm{e}$ shows the dependence of the group velocity $v_{g z}$ versus incident angle at the carrier frequency of $9.5 \mathrm{GHz}$. We see that $v_{g z}$ is positive at small incident angles ( $0-5$ degrees) and negative at larger incident angles. A negative group velocity as fast as $-0.3 \mathrm{c}$ is measured at $9.5 \mathrm{GHz}$ with an incident angle of 7.5 degrees.

\section{Discussion}

From Fig. 2, we see that the material loss, the anisotropy and the oblique incidence play indispensable roles in the generation of the ksurface distortion, which finally results in the anomalous dispersion. This particular k-surface distortion cannot appear in an isotropic medium, nor in the case with a normal incidence, which makes our approach differentiate itself from previously reported ones. However, causality still holds: in Fig. 3, both the front velocity and the group velocity (reflecting the phase velocity of the wave packets) are simultaneously illustrated, and we see that although the wave packets travel at a superluminal velocity, the information carried by them is not superluminally transmitted. In Fig. 4, both the frequency and the incidence angle dependence of the negative group velocity are demonstrated, which provides a new degree of freedom to engineer the dispersion property. It is also worth noting that, with an oblique incidence, the dispersion of group velocity would spread the wave packets not only in temporal axis, but also in spatial axes. At an incidence angle where the dispersion of group velocity is strong enough, e.g., at the critical angle $\theta_{c}$, multiple carriers modulated with different information can be spatially separated, and as such, it provides a potential approach for a temporal-spatial information processing.

To summarize, we have demonstrated the introducing of anomalous dispersion in a passive medium. We experimentally measured an unusual travelling of EM wave packets with a negative group velocity at the plasma frequency band, which is far away from the absorption resonance region. This abnormal wave packets propagation arises from the physical requirement of the dispersion continuity at the plasma frequency. Different from previously reported superluminal experiments, our approach does not require strong absorption resonance, and can be directly measured inside the medium. The proposed approach for engineering group indices in normal dispersive media is considerably easier than the existing methods. Introducing anomalous dispersion in a well-controlled manner would also greatly benefit the research of ultrafast photonics and is therefore suitable for diverse applications such as optical delay lines, optical data storage and devices for quantum information processing. Recent progress of active nanoplasmonic metamtaerials shows that active methods can be used to effectively compensate the metal losses, providing the possibility for fabricating low-loss optical uniaxial metamateirals in the future ${ }^{27,28}$.

\section{Methods}

Fabrication of the experimental sample and the retrieval of $\mu_{r z}$. The slab-like solidstate metamaterial sample consists of multilayer printed circuit boards (PCBs) carrying sub-wavelength split-ring resonators (SRRs). In the fabrication process, the metallic (copper) SRRs were printed on 0.5 -mm-thick FR4 substrates, with a relative permittivity around 4.6 at microwave frequencies. Each board contains 82 unit cells along the $\mathrm{x}$ direction with a periodicity of $6 \mathrm{~mm}$ and 15 unit cells in the $\mathrm{y}$ direction with a periodicity of $10 \mathrm{~mm}$. Eleven layers of such PCBs were aligned along the $\mathrm{z}$ direction spaced by $16 \mathrm{~mm}$ to form a slab-shaped sample with a width of $500 \mathrm{~mm}$ along the $\mathrm{x}$ direction, $150 \mathrm{~mm}$ in the $\mathrm{y}$ direction and a thickness of $160 \mathrm{~mm}$ in the $\mathrm{z}$ direction.

For an oblique incident wave with the electric field polarized in the y direction impinging upon such a medium, magnetic resonances can be induced at the metallic rings, yielding a strong dispersion of permeability with a negative band of $\mu_{r z}$. Meanwhile, there is no magnetic response in $\mathrm{x}$ and $\mathrm{y}$ directions, and thus $\mu_{r x}$ and $\mu_{r y}$ can be treated as unity. By utilizing the homogenization approach ${ }^{29,30}$, the dispersion of $\mu_{r z}$ can be retrieved from the scattering parameters from a full-wave simulation, and the result is shown in the inset of Fig. 3a. We see that the dispersion of $\mu_{r z}$ closely follows a classical Drude-Lorentz dispersion model: there is a strong absorption resonance around $9.19 \mathrm{GHz}$, and there is a typical plasma dispersion region between $9.35 \mathrm{GHz}$ and $9.65 \mathrm{GHz}$ (marked in gray) with $\omega_{p} \approx 9.45 \mathrm{GHz}$ and $\gamma_{e} \approx 0.05 \omega_{p}$. Different from the previous unity assumption, the retrieved $\varepsilon_{r}$ is around 2. Utilizing the retrieved $\mu_{r z}$ and $\varepsilon_{r}$, the transition zone of the k-surface distortion can be estimated as from $9.42 \mathrm{GHz}$ to $9.51 \mathrm{GHz}$ with a 15-degree incident angle. We note that, in the transition zone, the $\mu_{r z}$ is normal-dispersive and its imaginary part is quite small (varying from 0.02 to 0.2 ). As a comparison, the imaginary part is up to 7 in the conventional absorption resonance region).

Experimental setup. The experimental setup is shown in Fig. 3b. A $10 \mathrm{MHz}$ sinusoidal envelope signal was generated by a signal source (Agilent's 33210A) and used to modulate a carrier provided by a microwave source (Agilent's E8257D). The modulated signal was amplified and sent to a horn antenna located $150 \mathrm{~cm}$ away from the sample. The EM wave leaving from the antenna was electrically polarized along the $y$ direction, and impinged on the surface of the sample with an incident angle of 15 degrees. We placed the probe (a monopole antenna) at points 'a', 'b' and 'c', separately, to measure the intensity of the wave packets inside the sample. The detected signals from the probe were then demodulated by a crystal detector (Yamei Microwave's BO-20A) to recover the envelope signal. Triggered by the primitive envelope signal generated by the Agilent 33210A, the recovered envelope signal was displayed and sampled using a digital oscilloscope (Tektronix's MSO 4104B). To remove high frequency noise and waveform jitter, the built-in 20-MHz low pass filter and the average function of the MSO 4104B were enabled to obtain steady and clear envelope waveforms (see Supplementary Fig. S3 and S4).

1. Born, M., Wolf, E. \& Bhatia, A. B. Principles of optics: electromagnetic theory of propagation, interference and diffraction of light (Cambridge Univ. Pr.: Cambridge, 1999).

2. Jackson, J. D. Classical electrodynamics (Wiley: New York, 1999).

3. Brillouin, L. Wave propagation and group velocity (Academic Press: New York, 1960).

4. Bolda, E. L., Garrison, J. C. \& Chiao, R. Y. Optical pulse propagation at negative group velocities due to a nearby gain line. Physical Review A 49, 2938 (1994).

5. Bolda, E. L., Chiao, R. Y. \& Garrison, J. C. Two theorems for the group velocity in dispersive media. Physical Review A 48, 3890 (1993).

6. Chiao, R. Y. Superluminal (but causal) propagation of wave packets in transparent media with inverted atomic populations. Physical Review A 48, R34 (1993).

7. Chiao, R. Y. \& Boyce, J. Superluminahty, parelectricity, and karnshaw's theorem in media with inverted populations. Physical Review Letters 73, 3383(1994).

8. Faxvog, F. R. et al. Measured pulse velocity greater than $\mathrm{c}$ in a neon absorption cell. Appl. Phys. Lett. 17, 192 (1970).

9. Chu, S. \& Wong, S. Linear pulse propagation in an absorbing medium. Physical Review Letters 48, 738 (1982).

10. Dolling, G. et al. Simultaneous negative phase and group velocity of light in a Metamaterial. Science 312, 892-894 (2006).

11. Wang, L. J., Kuzmich, A. \& Dogariu, A. Gain-assisted superluminal light propagation. Nature 406, 277-279 (2000).

12. Stenner, M. D., Gauthier, D. J. \& Neifeld, M. A. The speed of information in a "fastlight" optical medium. Nature 425, 695-698 (2003).

13. Akulshin, A., Barreiro, S. \& Lezama, A. Steep anomalous dispersion in coherently prepared Rb vapor. Physical Review Letters 83, 4277 (1999).

14. Steinberg, A. M. \& Chiao, R. Y. Dispersionless highly superluminal propagation in a medium with a gain doublet. Physical Review A 49, 2071 (1994). 
15. Picholle, E., Montes, C., Leycuras, C., Legrand, O. \& Botineau, J. Observation of dissipative superluminous solitons in a brillouin fiber ring laser. Physical Review Letters 66, 1454 (1991).

16. Gehring, G. M., Schweinsberg, A., Barsi, C., Kostinski, N. \& Boyd, R. W. Observation of backward pulse propagation through a mediumwith a negative group velocity. Science 312, 895-897 (2006).

17. Zhang, L. et al. Superluminal propagation at negative group velocity in optical fibers based on brillouin lasing oscillation. Physical Review Letters 107, 093903 (2011).

18. Felsen, L. B. \& Marcuvitz, N. Radiation and Scattering of Waves (IEEE.: New York, 1994).

19. Eleftheriades, G. V. \& Balmain, K. G. Negative Refraction Metamaterials: Fundamental Principles and Applications (Wiley-IEEE.: New Jersey, 2005).

20. Guo, Y., Newman, W., Cortes, C. L. \& Jacob, Z. Applications of hyperbolic metamaterial substrates. Advances in Opto Electronics 2012, 1 (2012).

21. Krishnamoorthy, H. N. S., Jacob, Z., Narimanov, E., Kretzschmar, I. \& Menon, V. Topological transitions in metamaterials. Science 336, 205-209 (2012).

22. Landau, L. D. \& Lifshitz, E. M. Electrodynamics of Continuou Media (Pergamon Pr.: Oxford, 1984)

23. Milonni, P. W. Fast light, slow light and left-handed light. (CRC Press: Boca Raton, 2004).

24. Engheta, N. \& Ziolkowski, R. W. Metamaterials: Physics and Engineering Explorations (Wiley: New York, 2006).

25. Pendry, J. B. et al. Extremely low frequency plasmons in metallic mesostructures. Physical Review Letters 76, 4773 (1996).

26. Pendry, J. B. et al. Magnetism from conductors and enhanced nonlinear phenomena. IEEE Trans. Microwave Theory Tech. 47, 2075 (1999).

27. Meinzer, N. et al. Arrays of Ag split-ring resonators coupled to InGaAs singlequantum-well gain. Opt. Express 18, 24140 (2010).
28. Hess, O. et al. Active nanoplasmonic metamaterials. Nature material 11, 573 (2012).

29. Smith, D. R. et al. Determination of the effective permittivity and permeability of metamaterials from reflection and transmission coefficients. Physical Review B 65, 195104 (2002).

30. Chen, X. D. et al. Robust method to retrieve the constitutive effective parameters of metamaterials. Physical Review E 70, 016608 (2004).

\section{Acknowledgements}

This work is sponsored by NSFC under grants 61102003, 61071063 and 61131002 .

\section{Author contributions}

D.Y., G.Z., L.R. designed research; D.Y., J.W., Z.W., S.Q., J.H. performed research; all authors contributed to data interpretation and the composition of the paper. S.Q. supervised the project.

\section{Additional information}

Supplementary information accompanies this paper at http://www.nature.com/ scientificreports

Competing financial interests: The authors declare no competing financial interests.

License: This work is licensed under a Creative Commons

Attribution-NonCommercial-NoDerivs 3.0 Unported License. To view a copy of this license, visit http://creativecommons.org/licenses/by-nc-nd/3.0/

How to cite this article: Ye, D. et al. Negative Group Velocity in the Absence of Absorption Resonance. Sci. Rep. 3, 1628; DOI:10.1038/srep01628 (2013). 